\title{
Coloring fluorescence emission with silver nanowires
}

\author{
Timur Shegai, ${ }^{1, a)}$ Yingzhou Huang, ${ }^{2}$ Hongxing $\mathrm{Xu}^{2}{ }^{2}$ and Mikael Käll ${ }^{1}$ \\ ${ }^{1}$ Department of Applied Physics, Chalmers University of Technology, 41296 Göteborg, Sweden \\ ${ }^{2}$ Beijing National Laboratory for Condensed Matter Physics, Institute of Physics, Chinese Academy of \\ Sciences, Box 603-146, 100190 Beijing, People's Republic of China
}

(Received 11 February 2010; accepted 16 February 2010; published online 10 March 2010)

\begin{abstract}
We demonstrate that emission from Rhodamine-6G fluorophores adsorbed on silver nanowires experiences a spectral redshift upon propagation to the distal ends of the nanowire, with the shift being proportional to the propagation distance. The end of a nanowire thus constitutes a tunable fluorescence source controlled by a single easily adjustable parameter, i.e., the position of the excitation focal spot. The effect is made possible by a combination of radiatively undamped plasmon propagation and dispersive ohmic losses in the silver nanowire. The results may be important for the development of plasmonic waveguides, fast fluorescent color switches and various nanoscale fluorescence sensors. (C) 2010 American Institute of Physics. [doi:10.1063/1.3355545]
\end{abstract}

The control of fluorescence emission properties using metal nanoparticles is a challenging but increasingly important topic in nanophotonics and plasmonics. Crucial fluorophore properties, such as the excited state lifetime and the shape of the fluorescence spectrum, can be modified by plasmon resonances of metal nanostructures located in close proximity to the emitter. ${ }^{1-4}$ It was experimentally demonstrated that fluorescence can be enhanced, quenched, and spectrally shaped in various ways in the presence of plasmonic nanoparticles. ${ }^{5}$ Further progress includes enhancement of fluorescence resonance energy transfer ${ }^{6}$ and recently realized plasmonic based lasing in metal nanoparticles surrounded by a gain medium. ${ }^{7}$ However, it is difficult to achieve active control over fluorescence properties using metal nanoparticles because the fluorophore-metal interaction is based on localized surface plasmon resonances, predefined by passive parameters of the plasmonic system, that is the size, shape, and electromagnetic environment of the nanoparticle. On the other hand, active control over fluorescence emission could in principle be established by changing the distance between the emitter and the nanoparticle but this approach would require positioning with nanometer precision, which is difficult to realize in practice. ${ }^{5,8,9}$ In this Letter, we demonstrate an approach toward active fluorescence tuning that is free of these complications and based on using single crystalline silver nanowires. ${ }^{10}$

Silver nanowires support a number of subwavelength waveguide modes in the form of propagating surface plasmon polaritons (SPP's) and were demonstrated to act as Fabry-Perot resonators, giving rise to a standing wave modulation of scattering spectra collected from the wire ends. ${ }^{11}$ Although excitation of nanowire SPP's by a far-field source can be achieved only at discontinuities, i.e., at wire ends, ${ }^{11}$ near-field excitation, for example via interaction with excitons from quantum dot emitters or semiconductor nanowires, can occur anywhere along a wire. ${ }^{12-17}$ The propagating nature of the nanowire surface plasmons also manifests through the possibility of remote excitation of surfaceenhanced Raman scattering hot-spots. ${ }^{18}$ Here we show that

\footnotetext{
${ }^{\text {a) }}$ Author to whom correspondence should be addressed. Electronic mail: timurs@chalmers.se.
}

the emission spectrum generated by Rhodamine-6G (R6G) dye molecules and collected from the nanowire ends can be tuned by simply focusing the laser light at various positions along the wire. This result, which is based on the wavelength dependent ohmic losses in the nanowire system, could have practical significance in fields such as nano optical signal waveguiding, fast fluorescent color switches and development of nanoscale fluorescence sensors.

Single crystal silver nanowires were prepared using a wet chemistry method described by Sun and Xia ${ }^{10}$ The typical nanowire dimensions were 5-20 $\mu \mathrm{m}$ in length and 50$200 \mathrm{~nm}$ in diameter ${ }^{10}$ (see Fig. 1). The wires were attached to microscope glass slides $(25 \times 25 \mathrm{~mm})$ functionalized with polylysine $(0.25 \mathrm{mg} / \mathrm{ml}$ for $10 \mathrm{~min})$ by allowing them to adsorb for about $1 \mathrm{~min}$, after which the slide was thoroughly washed with Milli Q water and dried using compressed ni-

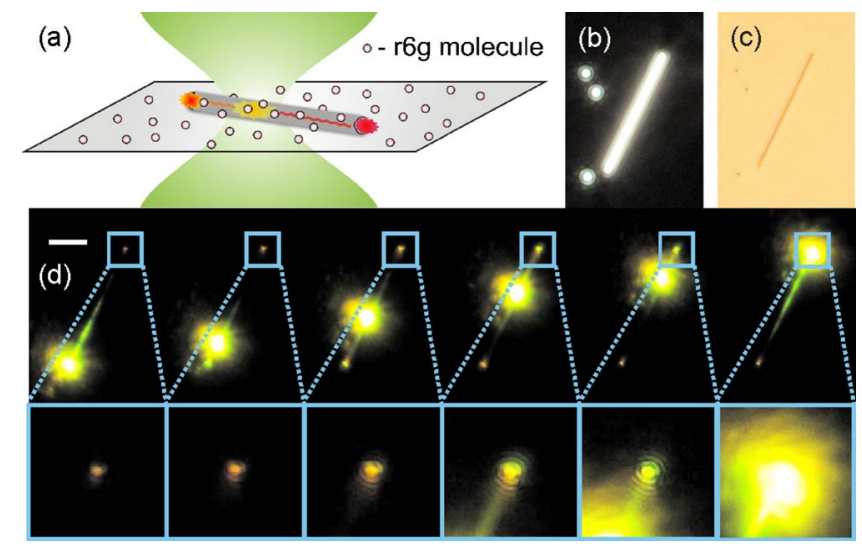

FIG. 1. (Color online) (a) Scheme of the experimental setup: A glass slide with an immobilized silver nanowire on top is covered with R6G molecules. The $532 \mathrm{~nm}$ laser beam is focused and positioned on the wire, exciting R6G molecules. Part of the fluorescence emission couple into plasmons propagating along the wire. The color of the emission at the distal ends of the wire depends on the propagation distance. Panels (b) and (c) show dark field and bright field optical images of the nanowire, respectively, while (d) shows fluorescence color images of the same nanowire as a function of laser spot position. The color of the emitting ends can be efficiently tuned from yellow-green to reddish by varying the position of the exciting beam (the most intense spot on the image). In the row at the bottom of panel (d) a magnified view of the region around the distal nanowire end outlined with a square is shown. The scale bar corresponds to $5 \mu \mathrm{m}$ length. 


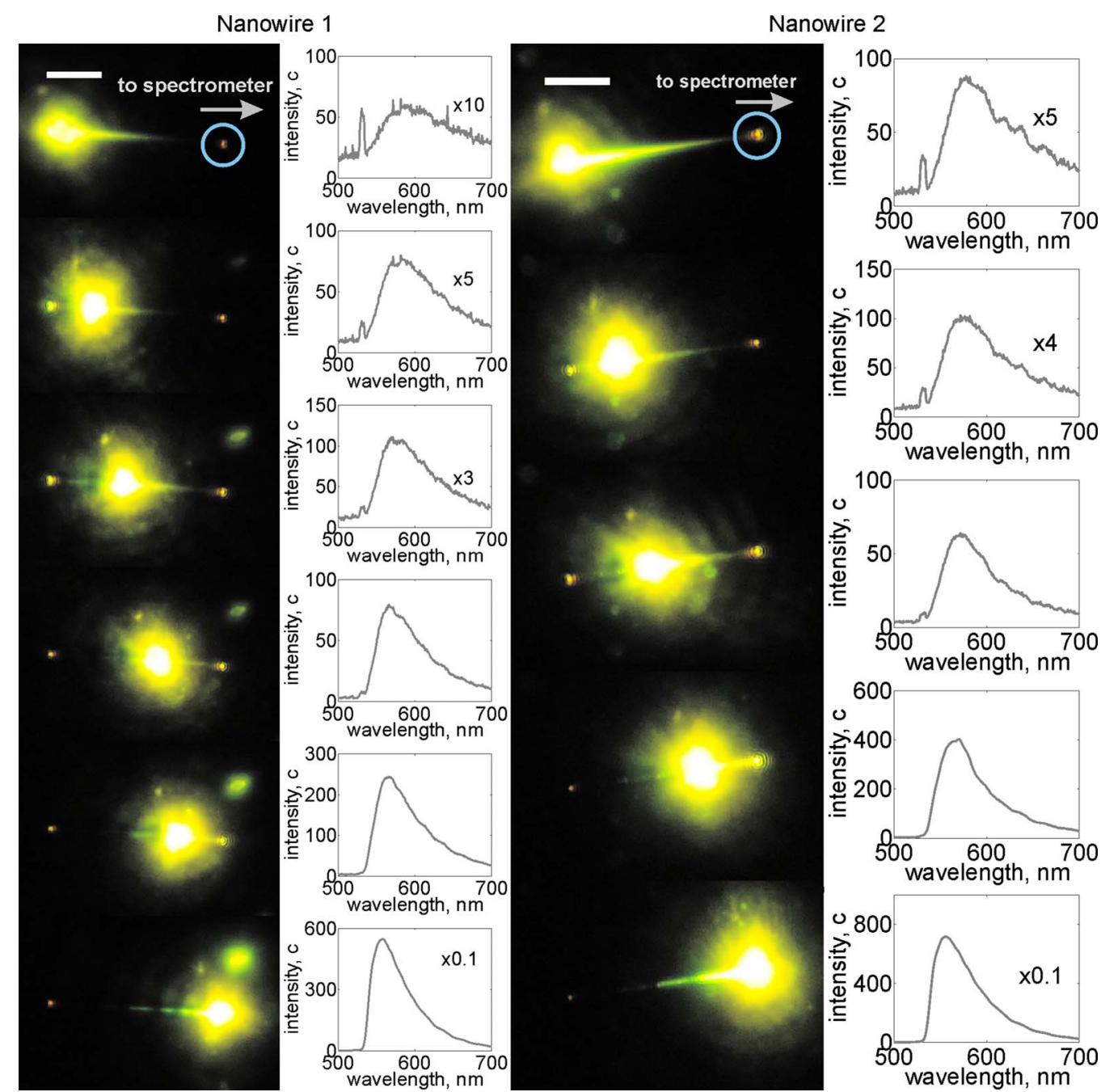

FIG. 2. (Color online) Fluorescence spectra collected from the right emitting end of the wire as a function of the laser spot position along the wire. The spectra were collected by means of a $400 \mu \mathrm{m}$ fiber aligned at the image plane of the microscope. The area from which the spectra were collected is outlined with the blue circle on the image. The white bar at the left upper corner corresponds to $5 \mu \mathrm{m}$ length.

trogen. This results in a sample of immobilized and randomly oriented silver nanowires covering the glass slide. We adsorbed a dense layer of R6G molecules on top of the Ag nanowires by simply covering the sample with an ethanol solution of the dye (a drop of $30 \mu \mathrm{l}$ of $10 \mu \mathrm{M}$ solution) and letting it dry completely. The Ag nanowire/R6G system was excited by a focused $532 \mathrm{~nm}$ laser beam (see Fig. 1) and the signal was collected through a Nikon Eclipse T2000-E inverted microscope equipped with a Nikon $100 \times$ NA $=0.7-1.3$ oil immersion objective and appropriate optical filters. Fluorescence images were registered by a Nikon D300s SLR camera.

As mentioned in the introduction, it is well known that incident light can be converted into propagating plasmons in the Ag nanowires via near field coupling. In the present case, near-field coupling is provided via the fluorophorenanowire interaction and the light being emitted from the wire ends originate in propagating plasmons excited at the focal spot. We found that the color of the emitting tip was sensitive to the position of the focal spot along the nanowire. This effect is shown in Fig. 1(d). As is seen from the figure, the color of R6G fluorescence emission exactly at the position of the focal spot is greenish-yellow, while the color of the emitting tip is redshifted. Moreover, the degree of red- shift is proportional to the distance between the excitation spot and the wire end, as is clearly seen in Fig. 1(d).

To further quantify this effect, we collected fluorescence spectra exclusively from the emitting tip as a function of the laser spot position along the wire. This is shown in Fig. 2. Here the spectra were collected from the right emitting tips of the wires by means of a fiber coupled spectrometer (Ocean Optics), with the fiber entrance being aligned to the emitting tip. The spectra show a clear modification of the fluorescence spectra (redshift and damping) with increasing distance between the excitation spot and the wire end. The reason for the fluorescence modification can be understood as follows: When fluorescent photons are converted into propagating plasmons, their initial spectrum is broad because the spectrum of R6G is rather broad. However, the plasmons experience losses upon propagation. In the case of silver nanowires, these losses are predominantly nonradiative but wavelength dependent. In particular, the propagation length corresponding to the green component of the fluorescent spectrum is much shorter than the propagation length of the red component, which means that the spectrum becomes more and more "reddish" with increasing propagation distance. For thin films, the propagation length can be estimated as, ${ }^{19}$ 

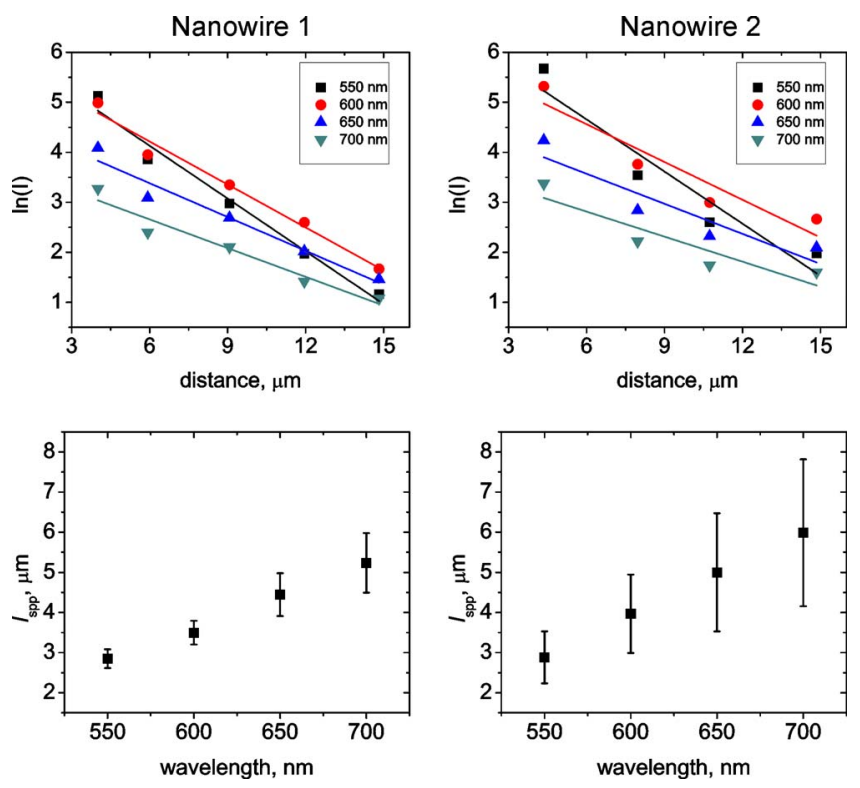

FIG. 3. (Color online) (top) Logarithm of fluorescence intensity as a function of distance between the excitation spot and the emitting wire end (from the data in Fig. 2) and corresponding linear fits. (bottom) Plasmon propagation length $\left(l_{\text {spp }}\right)$ for $550,600,650$, and $700 \mathrm{~nm}$ extracted from the fits.

$$
l_{\mathrm{spp}}=\frac{\lambda}{2 \pi} \times \frac{\varepsilon_{1}^{\prime}\left(\varepsilon_{1}^{\prime}+\varepsilon_{2}\right)}{\varepsilon_{1}^{\prime \prime} \varepsilon_{2}} \times \sqrt{\frac{\varepsilon_{1}^{\prime}+\varepsilon_{2}}{\varepsilon_{1}^{\prime} \varepsilon_{2}}},
$$

where $\lambda$ is the vacuum wavelength, $\varepsilon_{1}^{\prime}$ and $\varepsilon_{1}^{\prime \prime}$ are the real and imaginary parts of the metal dielectric function, and $\varepsilon_{2}$ is the dielectric function of the surrounding medium. By inserting the experimental values for the wavelength dependent dielectric function of silver, ${ }^{20}$ it is easy to see that the propagation length grows with vacuum wavelength, qualitatively confirming our results. It is clear, however, that the results for the nanowires are quantitatively different from the thin film formula (1). To show this, we extracted fluorescence intensity as a function of propagation distance for the two nanowires shown in Fig. 2 and fitted it to an exponential decay

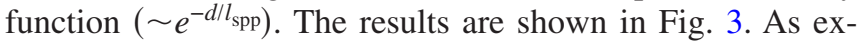
pected, the plasmon propagation length for the longer wavelength turns out to be longer than that for shorter ones. $l_{\mathrm{spp}}$ was extracted for 550,600, 650, and $700 \mathrm{~nm}$ with corresponding propagation lengths of about $3,4,5$, and $6 \mu \mathrm{m}$, and slight differences between nanowire 1 and 2. Ditlbacher et al. ${ }^{11}$ report a propagation length of about $10 \mu \mathrm{m}$ for a vacuum wavelength of $785 \mathrm{~nm}$, which is slightly longer than what can be expected from the trend in Fig. 3. Note, however, that we assumed a simple model for the plasmon propagation, not considering multiple reflections from the wire ends as well as additional losses due to the fact the whole wire is covered with R6G molecules. The former approximation should not introduce much of an error as long as the wire length is longer than the propagation length (which is the case for both wires shown in Fig. 2). However, the latter could introduce errors, especially in the green part of the spectrum, where R6G absorption is significant. In fact, it seems likely that this secondary absorption process can serve as an additional channel of "red" enrichment of the fluorescence signal propagating to the wire end.

A spectroscopic redshift upon surface plasmon propagation in nanowires has also been observed by Fedutik et al. ${ }^{12}$ and very recently by Yan et al. ${ }^{15}$ We thus can compare the propagation lengths with the data from Ref. 15, which turns out to be about 6 and $11 \mu \mathrm{m}$ for 532 and $650 \mathrm{~nm}$, respectively. This is slightly longer than both the results of the present study and the data of Ref. 11. The differences could be explained by the presence of the substrate, variations in wire diameter used in different studies as well as due to the additional radiative and nonradiative losses introduced by R6G molecules in this work.

In conclusion, we have demonstrated a tunable coloring of fluorescence emission originating from R6G molecules adsorbed on $\mathrm{Ag}$ nanowires obtained by simply varying the position of the excitation focal spot along the wire. This result may find important for developing and improving applications such as plasmonic waveguides, fast color switches, fluorescent sensors, and in general suggests a way toward realization of active plasmonics devices.

${ }^{1}$ E. M. Purcell, Phys. Rev. 69, 37 (1946).

${ }^{2}$ H. X. Xu, X. H. Wang, M. P. Persson, H. Q. Xu, M. Käll, and P. Johansson, Phys. Rev. Lett. 93, 243002 (2004).

${ }^{3}$ P. Johansson, H. X. Xu, and M. Käll, Phys. Rev. B 72, 035427 (2005).

${ }^{4}$ A. Kinkhabwala, Z. F. Yu, S. H. Fan, Y. Avlasevich, K. Mullen, and W. E. Moerner, Nat. Photonics 3, 654 (2009).

${ }^{5}$ P. Anger, P. Bharadwaj, and L. Novotny, Phys. Rev. Lett. 96, 113002 (2006).

${ }^{6}$ F. Reil, U. Hohenester, J. R. Krenn, and A. Leitner, Nano Lett. 8, 4128 (2008).

${ }^{7}$ M. A. Noginov, G. Zhu, A. M. Belgrave, R. Bakker, V. M. Shalaev, E. E. Narimanov, S. Stout, E. Herz, T. Suteewong, and U. Wiesner, Nature (London) 460, 1110 (2009)

${ }^{8}$ J. Seelig, K. Leslie, A. Renn, S. Kuhn, V. Jacobsen, M. van de Corput, C. Wyman, and V. Sandoghdar, Nano Lett. 7, 685 (2007).

${ }^{9}$ T. H. Taminiau, F. D. Stefani, F. B. Segerink, and N. F. Van Hulst, Nat. Photonics 2, 234 (2008).

${ }^{10}$ Y. G. Sun and Y. N. Xia, Adv. Mater. 14, 833 (2002).

${ }^{11}$ H. Ditlbacher, A. Hohenau, D. Wagner, U. Kreibig, M. Rogers, F. Hofer, F. R. Aussenegg, and J. R. Krenn, Phys. Rev. Lett. 95, 257403 (2005).

${ }^{12}$ Y. Fedutik, V. Temnov, U. Woggon, E. Ustinovich, and M. Artemyev, J. Am. Chem. Soc. 129, 14939 (2007).

${ }^{13}$ A. V. Akimov, A. Mukherjee, C. L. Yu, D. E. Chang, A. S. Zibrov, P. R. Hemmer, H. Park, and M. D. Lukin, Nature (London) 450, 402 (2007).

${ }^{14}$ X. Guo, M. Qiu, J. M. Bao, B. J. Wiley, Q. Yang, X. N. Zhang, Y. G. Ma, H. K. Yu, and L. M. Tong, Nano Lett. 9, 4515 (2009).

${ }^{15}$ R. X. Yan, P. Pausauskie, J. X. Huang, and P. D. Yang, Proc. Natl. Acad. Sci. U.S.A. 106, 21045 (2009).

${ }^{16}$ A. L. Falk, F. H. L. Koppens, C. L. Yu, K. Kang, N. D. Snapp, A. V. Akimov, M. H. Jo, M. D. Lukin, and H. Park, Nat. Phys. 5, 475 (2009).

${ }^{17}$ H. Wei, D. Ratchford, X. Q. Li, H. X. Xu, and C. K. Shih, Nano Lett. 9, 4168 (2009).

${ }^{18}$ Y. R. Fang, H. Wei, F. Hao, P. Nordlander, and H. X. Xu, Nano Lett. 9, 2049 (2009).

${ }^{19}$ H. Raether, Surface Plasmons on Smooth and Rough Surfaces and on Gratings (Springer, Berlin, 1988).

${ }^{20}$ P. B. Johnson and R. W. Christy, Phys. Rev. B 6, 4370 (1972). 